\title{
Frequent copy number variations of PI3K/AKT pathway and aberrant protein expressions of PI3K subunits are associated with inferior survival in diffuse large B cell lymphoma
}

Wenli Cui ${ }^{1,2,3,4 \dagger}$, Ying Cai ${ }^{1,2,3 \dagger}$, Weige Wang ${ }^{1,2,3}$, Zebing Liu ${ }^{1,2,3}$, Ping Wei ${ }^{1,2,3}$, Rui Bi ${ }^{1,2,3}$, Weixiang Chen ${ }^{1,2,3}$, Menghong Sun ${ }^{1,2,3}$ and Xiaoyan Zhou ${ }^{1,2,3^{*}}$

\begin{abstract}
Background: It has been reported that the PI3K/AKT signaling pathway is activated in diffuse large B-cell lymphoma (DLBCL), PI3K constitutive activation plays a crucial role in PI3K/AKT pathway. However, the copy number variations (CNVs) of PI3K subunits on gene level remain unknown in DLBCL. Therefore, the aim of the study is to investigate the CNV of PI3K subunits and their relationship with clinicopathological features exploring the possible mechanism underlying of PI3K activation in DLBCL.
\end{abstract}

Methods: CNV of 12 genes in the PI3K/AKT pathway was detected by NanoString nCounter in 60 de novo DLBCLs and 10 reactive hyperplasia specimens as controls. Meanwhile, immunohistochemistry $(\mathrm{IHC})$ was performed to

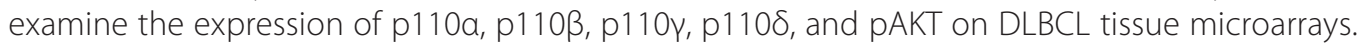

Results: All PI3K and AKT subunits, except for PIK3R1, had various CNVs in the form of copy number amplifications and copy number losses. Their rates were in the range of $8.3-20.0 \%$. Of them PIK3CA and PIK3CB gene CNVs were significantly associated with decreased overall survival $(P=0.029$ and $P=0.019$, respectively). IHC showed that the frequency of strong positive expression of p110a, p110 $\beta$, p110y, and $\mathrm{p} 110 \delta$ were $26.7 \%, 25.0 \%, 18.3 \%$, and $25.0 \%$ respectively, and they were found to be associated with decreased survival $(P=0.022, P=0.015, P=0.015$, and $P=0.008$, respectively). Expression of p110a was not only significantly associated with CNVs of PIK3CA $(P=0.002)$ but also positively correlated with strong positive expression of PAKT $(P=0.026)$.

Conclusions: CNV of PIK3CA is highly associated with aberrant p110a protein expression and subsequent activation of PI3KJAKT pathway. CNVs of PIK3CA and PIK3CB, and aberrant protein expression of p1 10 isoforms are of great important value for predicting inferior prognosis in DLBCL. Frequent CNVs of PI3K/AKT subunits may play an important role in the tumorigenesis of DLBCL.

Keywords: DLBCL, CNV, PI3K/AKT, Subunits, Survival

\footnotetext{
* Correspondence: xyzhou100@yahoo.com

${ }^{\dagger}$ Equal contributors

'Department of Pathology, Fudan University Shanghai Cancer Center, 270

Dong'an Road, Shanghai 200032, PR China

${ }^{2}$ Department of Oncology, Shanghai Medical College, Fudan University,

Shanghai 200032, PR China

Full list of author information is available at the end of the article
} 


\section{Background}

Diffuse large B-cell lymphoma (DLBCL) is the most common non-Hodgkin's lymphoma, accounting for $30-40 \%$ of adult non-Hodgkin's malignant lymphoma [1]. Although patients diagnosed with DLBCL are potentially curable with chemotherapy, the disease proves to be fatal in approximately $50 \%$ of patients [2]. Recently, given that there has been an increasing trend in the incidence of DLBCL, it is imperative to develop specific and effective treatments related to the pathogenesis of the disease.

Previous studies have reported that the phosphatidylinositol 3-kinase (PI3K) signaling pathway plays a critical role in regulating the growth and survival of DLBCL cells [3], and that constitutive phosphorylation of PI3K resulted in the activation of signaling that represented frequent events both for main pathway components and their downstream substrates [4]. Activated PI3K/AKT signaling pathways have been reported to be associated with decreased disease-free survival (DFS) and a poor response to treatment in patients with DLBCL [5]. This suggests that the PI3K/AKT pathway is potentially an important tumorigenic signaling route and an unfavorable prognostic factor in DLBCL.

PI3Ks consist of a large and complex family that contains three classes, I, II, and III. Of them, Class I PI3K is the most studied and plays a key role in the development and progression of tumors [6]. Class I contains the class IA catalytic subunits PIK3CA, PIK3CB, PIK3CD, and class IB catalytic subunit PIK3CG and the regulatory subunits PIK3R1, PIK3R2, and PIK3R3, while class II contains the catalytic subunits PIK3C2A, PIK3C2B, and PIK3C2G [4]. However, how each subunit precisely contributes to the progression and maintenance of tumors is largely undetermined. The PI3K/AKT signaling pathway can be activated by two main mechanisms: activating mutations and amplifications [7]. Amplification of genes encoding the catalytic subunits of PIK3CA, PIK3CB, PIK3CD, and PIK3CG has been reported in numerous solid tumors [7]. In lymphomas, PIK3CA has been reported to be amplified in 15/22 (68\%) cases of mantle cell lymphoma (MCL) [8], 9/161 (5.6\%) cases of chronic lymphocytic leukemia (CLL) [9], and mutated in 1/76 (1.3\%) cases of DLBCL [10]; while PIK3CD has been reported to be mutated in 3/73 (4.1\%) cases of DLBCL [11]. However, there have been few reports available regarding CNVs or mutations of other PI3K/AKT subunits and their contribution to the activation of the PI3K/AKT pathway in DLBCL.

In the present study, we focused mainly on the various PI3K/AKT subunits and profiled their CNVs using the NanoString nCounter assay and investigated their protein expression by immunohistochemistry (IHC). Furthermore, we analyzed the association of CNVs and protein expression with clinicopathological parameters in DLBCL. We also studied various members of the PI3K/AKT pathway simultaneously in the same set of DLBCL clinical samples as well as in a panel of lymphoma cell lines to investigate their involvement in the pathogenesis of DLBCL.

\section{Materials and methods}

Tissue specimens, cell lines, and patient information

A total of 70 fresh frozen samples including 60 DLBCLs and 10 lymph node reactive hyperplasias (RHs) were collected for CNV detection on the NanoString nCounter platform (NanoString Technologies, Seattle, WA, USA). DLBCL cell lines (DOHH2, OCI-Ly1, OCI-Ly8, and Toledo) and Burkitt's lymphoma (BL) cell lines (Raji, Namalwa) were included. Corresponding DLBCL formalin-fixed, paraffin-embedded (FFPE) tissues were collected for IHC detection of PI3K catalytic subunits. All cases used in the present study were retrieved during an 8-year period (2005-2012) from our tumor bank and the Department of Pathology in the Shanghai Cancer Center. Diagnoses were reviewed by two pathologists (Wenli Cui and Ying Cai) based on the 2010 World Health Organization classification. The other relevant clinical pathological information including primary site, B symptoms, bulky disease, performance status, lactate dehydrogenase (LDH) activity, stage, International Prognostic Index (IPI) were collected. The DLBCLs were classified into GCB and non-GCB subtype according to Hans algorithm [12]. 13/60 (22\%) patients received R-CHOP (Rituximab, cyclophosphamide, doxorubicin, vincristine, and prednisone) or R-CHOP-like therapy, and $47 / 60(78 \%)$ patients received CHOP or CHOP-like therapy. Patient clinical information was extracted from hospital records. Research protocols for this study were approved by the Ethics Committee at Fudan University Shanghai Cancer Center (Shanghai, China).

\section{NanoString nCounter assay}

Genomic DNA from fresh frozen tissue and cultured cell lines was extracted using a DNA extraction kit (Qiagen, Venlo, Netherlands) following the manufacturer's protocol. Only DNA samples with an OD A260/280 ratio between 1.7 and 1.9, which indicates optimal purity for DNA, were used for further study. For detection of CNVs in the PI3K/AKT pathway, a panel of customcompiled gene probes related to the pathway, including PI3K catalytic subunits PIK3CA, PIK3CB, PIK3CD, PIK3CG, PIK3C2A, PIK3C2B, and PIK3C2G, and regulated subunits PIK3R1 and PIK3R2, as well as AKT subunits AKT1, AKT2, and AKT3 were designed using NanoString nCounter technology and subsequently analyzed on the NanoString nCounter platform. NanoString probes were designed for the 12 genes according to different exons located in different regions (Table 1). Three 
Table 1 PI3K/AKT subunits, their localization and exons for probes

\begin{tabular}{lll}
\hline Genes & Localization & Exons for probes \\
\hline PIK3CA & $3 q 26.3$ & $9,10,20$ \\
PIK3CB & $3 q 22.3$ & $8,11,22$ \\
PIK3CD & $1 p 36.2$ & $1,4,10$ \\
PIK3CG & $7 q 22.3$ & $2,9,10$ \\
PIK3R1 & $5 q 13.1$ & $8,13,15$ \\
PIK3R2 & $19 q 13.2-q 13.4$ & $2,8,15$ \\
PIK3C2A & $11 p 15.5-p 14$ & $1,10,32$ \\
PIK3C2B & $1 \mathrm{q} 32$ & $3,14,35$ \\
PIK3C2G & $12 p 12$ & $2,16,26$ \\
AKT1 & $14 q 32.32$ & $2,4,13$ \\
AKT2 & $19 q 13.2$ & $4,5,11$ \\
AKT3 & $1 \mathrm{q} 44$ & $4,7,12$ \\
\hline
\end{tabular}

probes were designed for each gene. Each assay contained six positive dsDNA control probes, eight negative control probes, and 10 invariant control probes (INVs) designed for autosomal genomic regions predicted not to contain common CNVs.

The NanoString nCounter assay was performed according to NanoString's standard protocol. Briefly, $600 \mathrm{ng}$ of fragmented genomic DNA per assay was hybridized with the capture and reporter probes in $30 \mu \mathrm{L}$ total volume and incubated overnight at $65^{\circ} \mathrm{C}$ for at least $16 \mathrm{~h}$. The target and probe complexes were washed and immobilized in the cartridge. Genomic DNA was fragmented into small pieces (200-800 bp) and denatured to produce single strands. The custom CNV CodeSet was then hybridized to the fragmented denatured DNA sample in a single multiplexed reaction (up to 800 genomic loci per CodeSet). Hybridized DNA-CodeSet complexes were purified using the fully automated nCounter prep station, and reporters were counted using the nCounter digital analyzer. The data were normalized to the INVs and to positive and negative controls in each hybridization reaction. Finally, data analysis was performed using nSolver software.

Copy number was determined by averaging over three probes per region. If the average copy number was below 1.4, the gene was considered as one copy; if between 1.5 and 2.4, considered as two copies; and if between 2.5 and 3.4, considered as three copies, according to the manufacturer's protocol.

\section{Immunohistochemistry}

IHC was performed to detect expression of the four PI3K catalytic isoforms and pAKT on routinely processed FFPE specimens from which tissue microarrays (TMAs) were constructed using double cores (core diameter was $1 \mathrm{~mm}$ ) from selected areas of 60 cases of DLBCL and 10 cases of $\mathrm{RH}$. Briefly, serial sections from FFPE samples were collected onto poly-L-lysine coated slides and processed with a standard manual streptavidin-peroxidase technique using a biotin-free detection system (Dako, Carpinteria, CA, USA) after a heat-induced antigen retrieval procedure with EDTA for pAKT and citric acid for other antigens for 3 min. Primary antibodies were incubated with TMAs overnight. Omission of the primary antibody and its replacement with an antibody diluent were used as the negative control. A ready-to-use kit (EnVision ${ }^{\mathrm{Tm}}$; Dako) was used according to the manufacturer's instructions.

The source and dilution of the primary antibodies used were as follows: PI3K p110 $\alpha$ (\#4249, clone C73F8, 1:400), PI3K p110ß (\#3011, clone C33D4, 1:400), and PI3K p110Y (\#5405, clone D55D5, 1:200) were from Cell Signaling Technology (Beverly, MA, USA), PI3K p1108 (sc-7176, clone H-219, 1:200) was from Santa Cruz Biotechnology (Dallas, TX, USA), and pAKT (Ser 473) (\#2118-1, clone EP2109Y, 1:150) was from Epitomics (Burlingame, CA, USA).

\section{Scoring of immunostaining}

Expression of the four PI3K catalytic isoforms was evaluated blindly and independently by two pathologists (Wenli Cui and Ying Cai). The staining intensity was scored from 0 to 3: 0 , no appreciable staining in tumor cells (negative); 1 , slight staining in tumor cells (weak positive); 2: moderate staining in tumor cells (moderate positive); 3: distinct staining in tumor cells (strong positive) [5]. Cases were considered positive if $\geq 30 \%$ of the tumor cells were stained with an antibody, as described in a previous study involving IHC evaluation of TMAs [12].

\section{Statistical analysis}

Overall survival (OS) was measured from date of diagnosis to date of death of any cause, or latest follow-up. Survival analysis was performed using Kaplan-Meier survival curves with a statistical software package (IBM SPSS Statistics 20.0, Armonk, Town of North Castle, NY, USA) and compared with the use of logistic statistics (log-rank). The Fisher's exact or $\chi^{2}$ test was used for statistical analysis of categorical data. The Cox proportional hazard regression model was used for univariate analyses. A $P$-value $<0.05$ was considered statistically significant.

\section{Results}

CNV of 12 genes in the PI3K/AKT pathway in DLBCL

We analyzed CNVs of 12 genes in a total of 60 DLBCLs, six cell lines including DOHH2, OCI-Ly1, OCI-Ly8, Toledo, Raji, and Namalwa, and 10 lymphoid hyperplasias. The results showed that 11 of the 12 PI3K/AKT family members had significant CNVs, including copy number amplifications and copy number losses. These were PIK3CA (17\%), PIK3CB (20\%), PIK3CD (17\%), PIK3CG 
(8\%), PIK3C2A (8\%), PIK3C2B (13\%), PIK3C2G (20\%), PIK3R2 (23\%), AKT1 (18\%), AKT2 (18\%), and AKT3 (22\%) (Table 2). Among genes with CNVs, we found that the copy numbers of PIK3CA, PIK3CB, PIK3R2, and PIK3C2B were only amplified (10/10, 12/12, 14/14, and 8/8, respectively); copy numbers of PIK3CG, PIK3C2A, PIK3C2G, AKT1, AKT2, and AKT3 were amplified in the majority of cases $(4 / 5,4 / 5,11 / 12,7 / 11,8 / 11$, and $11 / 13$, respectively), whereas copy number losses occurred in fewer cases $(1 / 5,1 / 5,1 / 12,4 / 11,3 / 11$, and $2 / 13$, respectively); copy number losses for PIK3CD occurred in the majority of cases (7/10), whereas amplifications occurred in a few cases (3/10) (Table 3). In DLBCL, the CNV frequency of PI3K-AKT subunits was in the range of 1-10 genes; in one of the 60 DLBCLs, 10 different genes were detected with CNVs. In this sample set, $58.3 \%(35 / 60)$ of patients had at least one CNV and $48.3 \%(29 / 60)$ had at least two co-occurring CNVs in the component of PI3K/AKT subunit genes (Table 4). CNVs were identified in most of the PI3K/AKT family genes in DLBCL. There was no statistically correlation found between any CNVs of PI3K gene family and that of AKT gene family, indicating there was no cause-and-effect interplay between CNVs of PI3K and AKT.

PIK3CG was found to be amplified in all GCB cell lines, including DOHH2, OCI-Ly1, OCI-Ly8, and Toledo; PIK3C2A and AKT1 were amplified in OCI-Ly1, OCI-Ly8, and Toledo; while PIK3C2B and PIK3C2G were amplified only in Toledo (Table 5).

Protein expression of PI3K catalytic subunits in DLBCL To further investigate the association between CNVs of PI3K subunits and their protein expression, and the

Table 2 The frequency CNV of PI3K/AKT subunits in 60 DLBCLs by NanoString nCounter analysis

\begin{tabular}{lllll}
\hline Gene & $\begin{array}{l}\text { Case } \\
\text { no. }\end{array}$ & $\begin{array}{l}\text { Copy number } \\
\text { amplification } \\
\text { no. (\%) }\end{array}$ & $\begin{array}{l}\text { Copy number } \\
\text { loss no. (\%) }\end{array}$ & $\begin{array}{l}\text { Total CNV } \\
\text { frequency } \\
\text { no. (\%) }\end{array}$ \\
\hline PIK3CA & 60 & $10(17)$ & $0(0)$ & $10(17)$ \\
PIK3CB & 60 & $12(20)$ & $0(0)$ & $12(20)$ \\
PIK3CD & 60 & $3(5)$ & $7(12)$ & $10(17)$ \\
PIK3CG & 60 & $4(6)$ & $1(2)$ & $5(8)$ \\
PIK3R1 & 60 & $0(0)$ & $0(0)$ & $0(0)$ \\
PIK3R2 & 60 & $14(23)$ & $0(0)$ & $14(23)$ \\
PIK3C2A & 60 & $4(6)$ & $1(2)$ & $5(8)$ \\
PIK3C2B & 60 & $8(13)$ & $0(0)$ & $8(13)$ \\
PIK3C2G & 60 & $11(18)$ & $1(2)$ & $12(20)$ \\
AKT1 & 60 & $7(12)$ & $4(6)$ & $11(18)$ \\
AKT2 & 60 & $8(13)$ & $3(5)$ & $11(18)$ \\
AKT3 & 60 & $11(18)$ & $2(3)$ & $13(22)$ \\
\hline
\end{tabular}

significance of Class I subunits in DLBCL, we analyzed the expression of $\mathrm{p} 110 \alpha, \mathrm{p} 110 \beta, \mathrm{p} 110 \gamma, \mathrm{p} 110 \delta$, and pAKT protein in a TMA of 60 DLBCLs using IHC. All of these proteins were expressed in the cytoplasm. In DLBCL, proteins were diffusely expressed in tumor cells, while in RH they were locally expressed in germinal centers (Figure 1). The expression frequencies of $\mathrm{p} 110 \alpha, \mathrm{p} 110 \beta, \mathrm{p} 110 \gamma, \mathrm{p} 110 \delta$, and $\mathrm{pAKT}$ protein were $80 \%, 81.6 \%, 81.6 \%, 81.6 \%$, and $75 \%$, respectively. Strong positive expression of the above proteins was found in $26.7 \%, 25.0 \%, 25.0 \%, 18.3 \%$, and $16.7 \%$ of cases, respectively. Among the four PI3K subunit proteins expressed, only $\mathrm{p} 110 \alpha$ showed strong positive expression, which was positively correlated with CNVs of PIK3CA ( $P=$ $0.002)$. P110 $\alpha$ strong positive expression was also correlated with strong positive expression of pAKT $(P=$ 0.026). Other strong positive expressions of $\mathrm{p} 110 \beta$, $\mathrm{p} 110 \gamma$, and $\mathrm{p} 110 \delta$ have no correlation with CNVs of PIK3CB, PIK3CG and PIK3CD. There was no significant correlation between the expression of these p110 isoforms and expression of pAKT.

\section{Association between CNVs in PI3K/AKT genes and clinicopathological characteristics in DLBCL}

Among the 60 patients with DLBCLs, their ages were in the range of 21-86 years with a mean age of 58 years. Fifty-seven cases had follow-up data from 2 to 79 months, with the average period being 34 months. During this period, $15 / 57$ (26\%) patients died. There was a significant association of shorter survival with CNVs of PIK3CA and PIK3CB (Figure 2). Patients with CNVs of PIK3CA and PIK3CB had significantly shorter survival times respectively $(P=0.029, \mathrm{n}=10 ; P=0.019, \mathrm{n}=12)$ than those with two wild-type copies (Figure 2A, Figure 2B). Patients whose DLBCLs had either PIK3CA or PIK3CB CNVs had significantly shorter survival times $(P=0.007, \mathrm{n}=13)$ than those without CNVs (Figure 2E). Both PIK3CA and PIK3CB CNVs had no significant shorter survival times $(P=0.069, \mathrm{n}=8)$ than those without CNVs (Figure 2F). No significant differences or similarities in survival were seen for patients with CNVs of PIK3CD (Figure 2C), PIK3CG (Figure 2D), PIK3C2A, PIK3C2B, PIK3C2G, PIK3R2, AKT1, AKT2, or AKT3. CNVs of PIK3CA and PIK3CB were higher in the non-GCB DLBCLs $(n=8$ and $n=10$, respectively) than in the GCB DLBCLs $(n=2$ and $n=2$, respectively). No difference in different pathological types was seen in other subunits. There were no significant differences between CNVs of PIK3CA, PIK3CB, PIK3CD, and PIK3CG with clinicopathological characteristics, including sex, age, primary site, B symptoms, bulky disease, performance status, LDH activity, stage, IPI, or pathological type (Table 6). Clinicopathological characteristics had no impact on survival through Cox regression univariate analysis. 
Table 3 The distribution of varied CNV in each PI3K/AKT subunits by NanoString nCounter analysis

\begin{tabular}{|c|c|c|c|c|c|c|}
\hline \multirow[t]{2}{*}{ Gene } & \multirow[t]{2}{*}{ Total CNV no. } & \multirow{2}{*}{$\begin{array}{l}\text { Copy number loss no. (\%) } \\
1 \text { copy }\end{array}$} & \multicolumn{3}{|c|}{ Copy number amplification no. (\%) } & \multirow{2}{*}{$\begin{array}{l}\text { Total copy number } \\
\text { amplification no. }(\%)\end{array}$} \\
\hline & & & 3 copy & 4 copy & 6 copy & \\
\hline PIK3CA & 10 & $0(0)$ & $9(90)$ & $1(10)$ & $0(0)$ & $10(100)$ \\
\hline PIK3CB & 12 & $0(0)$ & $11(92)$ & $1(8)$ & $0(0)$ & $12(100)$ \\
\hline PIK3CD & 10 & $7(70)$ & $2(20)$ & $1(10)$ & $0(0)$ & $0(30)$ \\
\hline PIK3CG & 5 & $1(20)$ & $4(80)$ & $0(0)$ & $0(0)$ & $4(80)$ \\
\hline PIK3R1 & 0 & $0(0)$ & $0(0)$ & $0(0)$ & $0(0)$ & $0(0)$ \\
\hline PIK3R2 & 14 & $0(0)$ & $12(86)$ & $1(7)$ & $1(7)$ & $14(100)$ \\
\hline $\mathrm{PIK} 3 \mathrm{C} 2 \mathrm{~A}$ & 5 & $1(20)$ & $4(80)$ & $0(0)$ & $0(0)$ & $4(80)$ \\
\hline PIK3C2B & 8 & $0(0)$ & $8(100)$ & $0(0)$ & $0(0)$ & $8(100)$ \\
\hline PIK3C2G & 12 & $1(8)$ & $10(84)$ & $1(8)$ & $0(0)$ & $11(92)$ \\
\hline AKT1 & 11 & $4(36)$ & $6(55)$ & $1(9)$ & $0(0)$ & $7(64)$ \\
\hline AKT2 & 11 & $3(27)$ & $8(73)$ & $0(0)$ & $0(0)$ & $8(73)$ \\
\hline AKT3 & 13 & $2(15)$ & $9(69)$ & $2(16)$ & $0(0)$ & $11(85)$ \\
\hline
\end{tabular}

Association between protein expression of PI3K catalytic subunits and clinicopathological features of DLBCL

There were no positive correlations between strong positive expression of $\mathrm{p} 110 \alpha, \mathrm{p} 110 \beta, \mathrm{p} 110 \gamma$, and $\mathrm{p} 110 \delta$ with clinicopathological characteristics, including sex, age, primary site, B symptoms, bulky disease, performance status, LDH activity, stage, IPI, and pathological type, except for $\mathrm{p} 110 \delta$, which had a significant difference in high IPI $(P=0.037)$ (Table 7$)$. Strong positive expression of $\mathrm{p} 110 \alpha, \mathrm{p} 110 \beta, \mathrm{p} 110 \gamma$, and $\mathrm{p} 110 \delta$ was found to be associated with decreased survival $(P=0.022, P=0.015$, $P=0.015$, and $P=0.008$ respectively) (Figure $3 \mathrm{~A}, 3 \mathrm{~B}$, $3 C, 3 D)$. Strong and moderate expression of pAKT associated with decreased survival $(P=0.165)$ (Figure 3E).

\section{Discussion}

Given the important involvement of the PI3K/AKT pathway in the pathogenesis of tumors, and given the paucity of datas regarding $\mathrm{CNV}$ in PI3K/AKT gene members in DLBCL, we first investigated CNVs using

Table 4 CNV of PI3K/AKT subunits genes involved in 60 DLBCLs

\begin{tabular}{lllll}
\hline & Genes involoved $(\geq)$ & Total case no. & CNV no. & Frequency \\
\hline 1 genes & 60 & 35 & $58 \%$ \\
2 genes & 60 & 29 & $48 \%$ \\
3 genes & 60 & 25 & $42 \%$ \\
& 4 genes & 60 & 18 & $30 \%$ \\
PI3KAKT & 5 genes & 60 & 12 & $20 \%$ \\
subunits & 6 genes & 60 & 6 & $10 \%$ \\
& 7 genes & 60 & 3 & $5 \%$ \\
& 9 genes & 60 & 2 & $3 \%$ \\
& 10 genes & 60 & 1 & $2 \%$ \\
\hline
\end{tabular}

NanoString nCounter's method [13] in 12 members of the PI3K/AKT signaling pathway in human DLBCL using an nCounter $\mathrm{CNV}$ assay. It was found that all PI3K and AKT subunits besides PIK3R1 had CNVs to a different extent, generally, with the frequency ranging from $8.3 \%$ to $23 \%$. Among which, CNVs of PIK3CA and PIK3CB were significantly associated with inferior overall survival. Additionally, protein expression of p110 $\alpha$, $\mathrm{p} 110 \beta, \mathrm{p} 110 \gamma$, and $\mathrm{p} 110 \delta$ using IHC method were also found to be associated with decreased survival.

CNVs have been found in some genes that are components of the PI3K/AKT pathway in various tumors [14-16]. However, little has been reported about CNVs of the PI3K/AKT in DLBCL. Here we have for the first time detected CNVs in almost all members of the PI3K/

Table 5 Copy number of PI3K/AKT pathway in DLBCLs and BLs cell lines

\begin{tabular}{|c|c|c|c|c|c|c|}
\hline & \multicolumn{4}{|c|}{ DLBCL } & \multicolumn{2}{|r|}{$B L$} \\
\hline & $\mathrm{DOHH} 2$ & OCI-LY1 & OCI-LY8 & Toledo & Raji & Namalwa \\
\hline PIK3CA & 2 & 2 & 2 & 2 & 2 & 2 \\
\hline PIK3CB & 2 & 2 & 2 & 2 & 2 & 2 \\
\hline PIK3CD & 2 & 2 & 2 & 2 & 2 & 2 \\
\hline PIK3CG & 3 & 3 & 3 & 3 & 2 & 2 \\
\hline PIK3R1 & 2 & 2 & 2 & 2 & 2 & 2 \\
\hline PIK3R2 & 2 & 2 & 2 & 2 & 2 & 2 \\
\hline PIK3C2A & 2 & 3 & 3 & 3 & 2 & 2 \\
\hline PIK3C2B & 2 & 2 & 2 & 3 & 2 & 2 \\
\hline PIK3C2G & 2 & 2 & 2 & 3 & 2 & 2 \\
\hline AKT1 & 2 & 3 & 3 & 3 & 2 & 2 \\
\hline AKT2 & 2 & 2 & 2 & 2 & 2 & 2 \\
\hline AKT3 & 2 & 2 & 2 & 4 & 2 & 2 \\
\hline
\end{tabular}




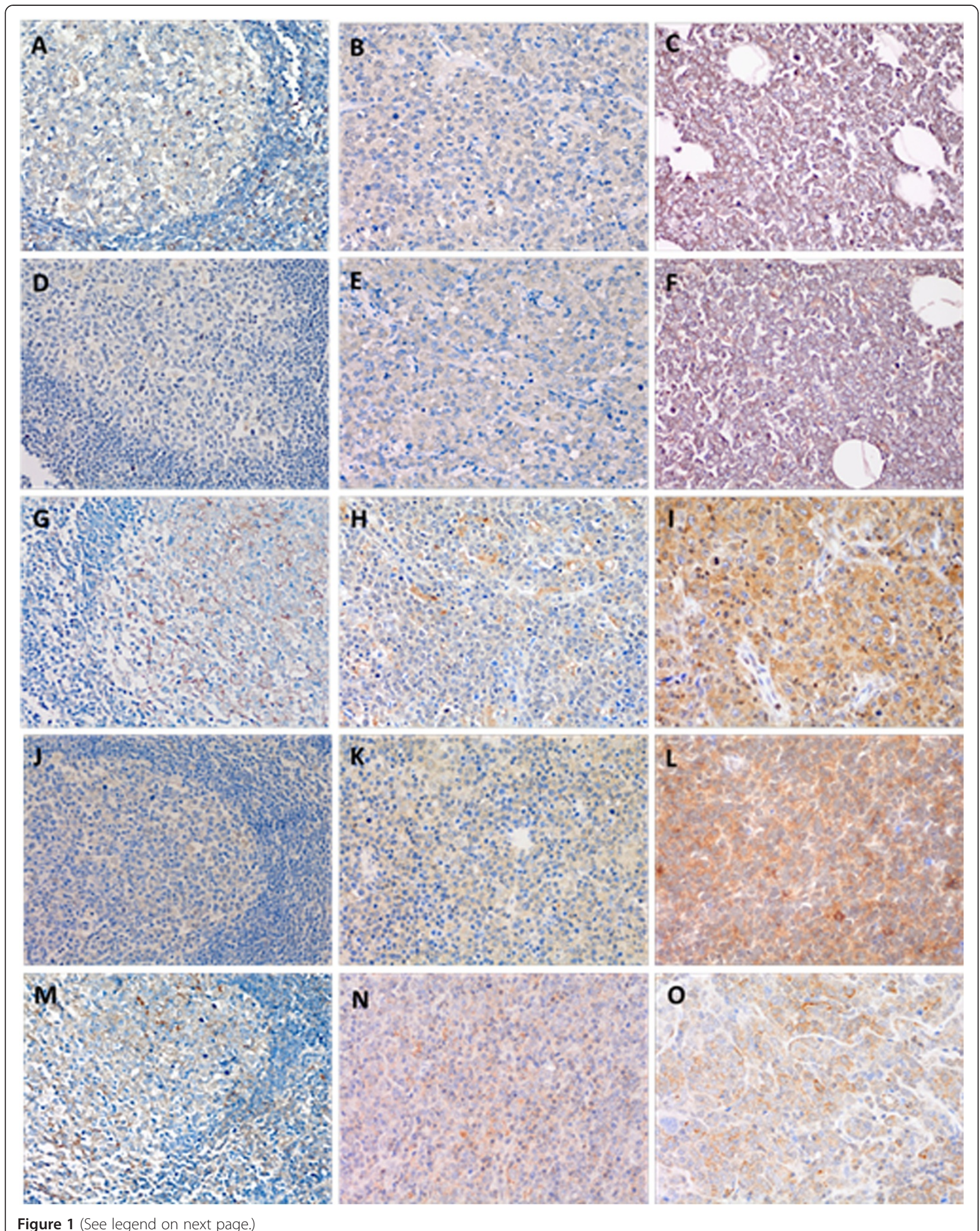


(See figure on previous page.)

Figure 1 Protein expression of $\mathrm{p} 110 a, \mathrm{p} 110 \beta, \mathrm{p} 110 \gamma, \mathrm{p} 110 \delta$, and $\mathrm{pAKT}$ was performed on tissue microarrays by Immunohistochemistry (IHC). A-C. Weak positive of germinal center in $\mathrm{RH}$, weak positive and strong positive of p110a in DLBCL D-F. Weak positive of germinal center in $\mathrm{RH}$, weak positive and strong positive of $\mathrm{p} 110 \beta$ in DLBCL G-I. Weak positive of germinal center in RH, weak positive and strong positive of p110y in DLBCL J-L. Weak positive of germinal center in $\mathrm{RH}$, weak positive and strong positive of p1108 in $\mathrm{DLBCL} \mathbf{M}-\mathbf{O}$. Weak positive of germinal center in $\mathrm{RH}$, weak positive and strong positive of PAKT in DLBCL.

AKT signaling pathway in DLBCL. We have revealed CNVs profiles and genetic abnormality as common event in the PI3K/AKT signaling pathway in DLBCL, providing evidence and possible explanation for the pathogenesis of DLBCL on genetic level.

There have been reports showed that PIK3CA gene was happened to CNVs in ovarian cancer [17], cervical cancer [18], and gastric cancer [19]; and that CNVs in PIK3CA were reported to be an independent factor for predicting poor OS of patients with ovarian cancers [17] and gastric cancers [19]. Our result showed that each CNVs of PIK3CA and PIK3CB has significant shorter survival. Either CNVs of PIK3CA or PIK3CB had significant shorter survival too, indicating either PIK3CA or PIK3CB has significant effects on overall survival. Both
CNVs of PIK3CA and PIK3CB had the effect trend on survival effect, there was no statistically significance.

In an earlier study, the authors discovered that amplification of PIK3CD was significantly higher in patients who were sensitive to rituximab than that in patients who were resistant to rituximab [20], suggesting that CNVs of PIK3CD may be a useful marker used for chemotherapy sensitivity when selecting the appropriate chemotherapeutic treatment for patients with DLBCL. Alizadeh et al. found that PIK3CG was highly expressed in GCB (germinal center B-cell like), and patients with GCB DLBCL had a significantly better OS than those with $\mathrm{ABC}$ (activated B-cell like) DLBCL [21], which was consistent with our detection that PIK3CG expression was significantly higher in GCB cell lines $(n=4)$.

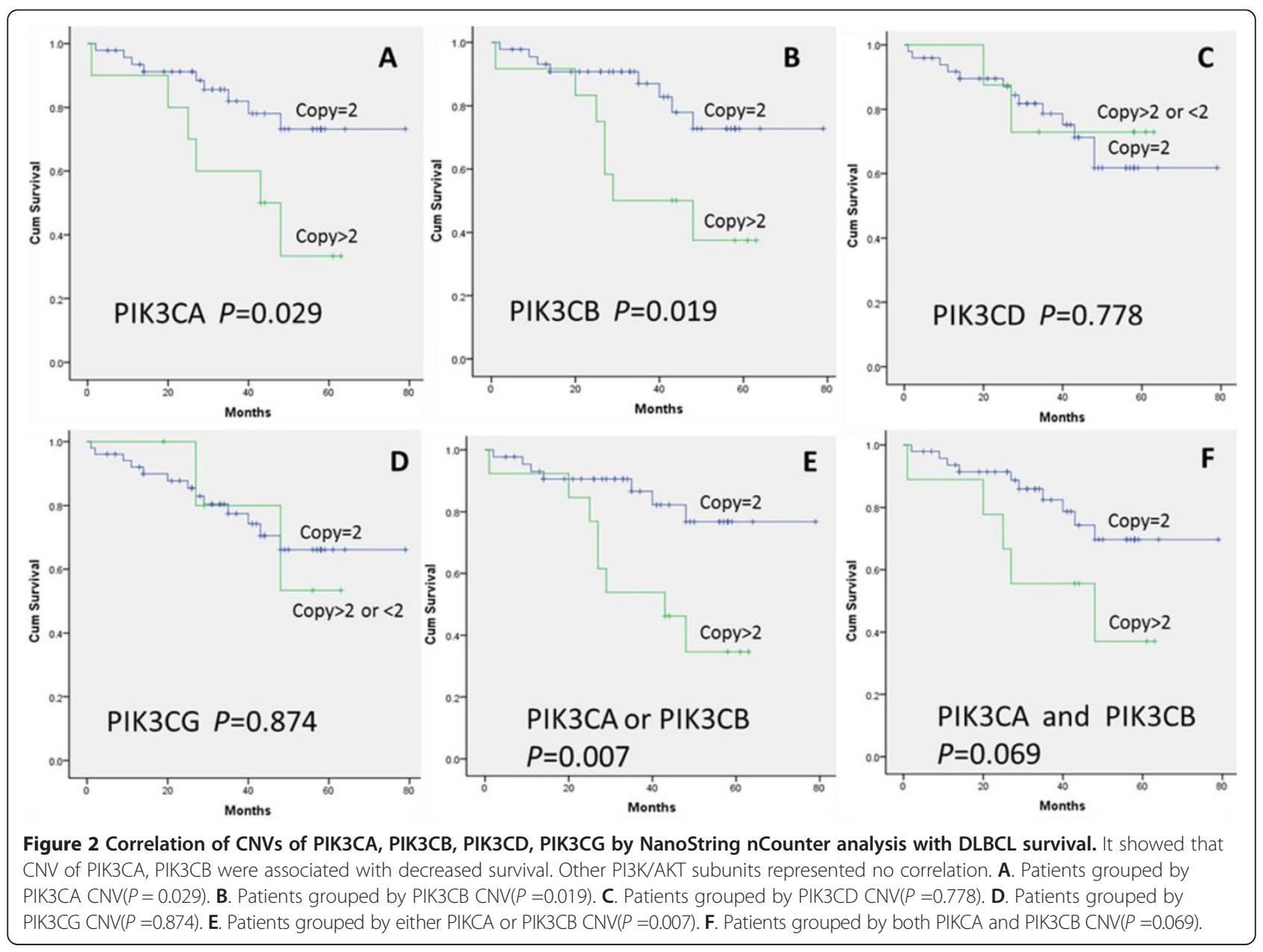


Table 6 CNV of PI3K subunits in 60 patients with DLBCLs by Nanostring nCounter analysis

\begin{tabular}{|c|c|c|c|c|c|c|c|c|c|}
\hline & & PIK3CA & $P$ value & PIK3CB & $P$ value & PIK3CD & $P$ value & PIK3CG & $P$ value \\
\hline Total no. of patients & 60 & & & & & & & & \\
\hline \multicolumn{10}{|l|}{ Sex } \\
\hline Male & 39 & $5(12.8)$ & 0.298 & $7(17.9)$ & 0.737 & $10(25.6)$ & 0.011 & $5(12.8)$ & 0.412 \\
\hline Female & 21 & $5(23.8)$ & & $5(23.8)$ & & $0(0)$ & & $1(4.8)$ & \\
\hline \multicolumn{10}{|l|}{ Age, years } \\
\hline$<60 y$ & 36 & $6(16.7)$ & 1.000 & $5(13.9)$ & 0.193 & $6(16.7)$ & 1.000 & $5(13.9)$ & 0.387 \\
\hline$\geq 60 y$ & 24 & $4(16.7)$ & & $7(29.2)$ & & $4(16.7)$ & & $1(4.2)$ & \\
\hline \multicolumn{10}{|l|}{ Primary site } \\
\hline node & 54 & $10(18.5)$ & 0.557 & $11(20.4)$ & 1.000 & $9(16.7)$ & 1.000 & $6(11.1)$ & 1.000 \\
\hline Perpheral node & 6 & $0(0)$ & & $1(16.7)$ & & $1(16.7)$ & & $0(0.0)$ & \\
\hline \multicolumn{10}{|l|}{ B symptoms } \\
\hline Absence & 37 & 7 (18.9) & 0.727 & $7(18.9)$ & 1.000 & $6(16.2)$ & 1.000 & $2(5.4)$ & 0.191 \\
\hline Presence & 23 & $3(13.0)$ & & $5(21.7)$ & & $4(17.4)$ & & $4(17.4)$ & \\
\hline \multicolumn{10}{|l|}{ Bulky disease } \\
\hline$<10 \mathrm{~cm}$ & 47 & $7(14.9)$ & 0.506 & $8(17.0)$ & 0.552 & $7(14.9)$ & 1.000 & $4(8.5)$ & 0.347 \\
\hline$\geq 10 \mathrm{~cm}$ & 4 & $1(25.0)$ & & $1(25.0)$ & & $0(0)$ & & $1(25.0)$ & \\
\hline \multicolumn{10}{|l|}{ Performance status } \\
\hline ECOG 0-1 & 50 & $8(16.0)$ & 1.000 & $8(16.0)$ & 1.000 & $7(14.0)$ & 0.125 & $6(12.0)$ & 1.000 \\
\hline ECOG 2-4 & 4 & $0(0.0)$ & & $1(25.0)$ & & $2(50.0)$ & & $0(0)$ & \\
\hline \multicolumn{10}{|l|}{ LDH } \\
\hline$<2 *$ insitutioal ULN & 30 & $6(34.9)$ & 0.715 & $6(20.0)$ & 1.000 & $6(20.0)$ & 0.117 & $3(10.0)$ & 1.000 \\
\hline$>2 *^{*}$ nsitutioal ULN & 24 & $3(12.5)$ & & $5(20.8)$ & & $1(4.2)$ & & $3(12.5)$ & \\
\hline \multicolumn{10}{|l|}{ Extranodal site } \\
\hline$\leq 1$ & 50 & $9(18.0)$ & 1.000 & $11(22.0)$ & 0.571 & $7(14.0)$ & 1.000 & $6(12.0)$ & 1.000 \\
\hline$>1$ & 4 & $0(0)$ & & $0(0.0)$ & & $0(0)$ & & $0 / 4(0)$ & \\
\hline \multicolumn{10}{|l|}{ Stage } \\
\hline$|/| \mid$ & 29 & $3(10.3)$ & 0.161 & $3(10.3)$ & 0.087 & $4(13.8)$ & 1.000 & $1(3.4)$ & 0.076 \\
\hline III/IV & 23 & $6(26.1)$ & & $7(30.4)$ & & $3(13.0)$ & & $5(21.7)$ & \\
\hline \multicolumn{10}{|l|}{$\mid \mathrm{PI}$} \\
\hline $0-1$ & 30 & $5(16.7)$ & 1.000 & $4(13.3)$ & 0.198 & $4(13.3)$ & 1.000 & $4(13.3)$ & 0.678 \\
\hline $2-5$ & 25 & $4(16.0)$ & & $7(28.0)$ & & $4(16.0)$ & & $2(8.0)$ & \\
\hline \multicolumn{10}{|l|}{ Pathology } \\
\hline GCB & 26 & $2(76.9)$ & 0.163 & $3(11.5)$ & 0.152 & $5(19.2)$ & 0.733 & $3(11.5)$ & 1.000 \\
\hline non-GCB & 34 & $8(23.5)$ & & $9(26.5)$ & & $5(14.7)$ & & $3(8.8)$ & \\
\hline
\end{tabular}

$D L B C L$ diffuse large B-cell lymphoma, GCB germinal center B cell, IPI international prognostic index, $L D H$ lactate dehydrogenase.

Among all of the members that we analyzed, the CNVs of PIK3CA and PIK3CB were positively and significantly associated with prognosis compared with other members. Depending on previous research, non-GCB represented poor overall survival than GCB. Although CNVs of PIK3CA and PIK3CB were apparently higher in non-GCB group $(80 \%(8 / 10)$ and $67 \%(8 / 12)$, respectively) than that in GCB group, there was no statistically significant. Copy number amplification of either PIK3CA or PIK3CB located in the same region (3q) showed a correlation with poor survival in DLBCL patients, indicating that this region of amplification has significant correlation with survival in DLBCL. There was no significant correlation between the CNV and protein level other than PIK3CA, indicating that $\mathrm{CNV}$ of these genes might partially contribute to the aberrant expression of PI3K isoforms supposedly. The inconsistency we discovered here is highly similar to an earlier report by Ye ZQ and colleagues whose finding is that there was inconsistency between $\mathrm{CNV}$ and protein differential expression for the most genes [22]. 
Table 7 Strong positive expresssion of p110 isforms and pAKT in 60 patients with DLBCLs by IHC

\begin{tabular}{|c|c|c|c|c|c|c|c|c|c|c|c|}
\hline & & p110a & $P$ value & p110 & $P$ value & p1108 & $P$ value & p110y & $P$ value & pAKT & $P$ value \\
\hline Total no. of patients & 60 & & & & & & & & & & \\
\hline \multicolumn{12}{|l|}{ Age, years } \\
\hline$<60 y$ & 36 & $12(33.3)$ & 0.037 & $10(27.8)$ & 0.639 & $12(33.3)$ & 0.068 & $7(19.4)$ & 1.000 & $7(19)$ & 0.293 \\
\hline$\geq 60 y$ & 24 & $4(16.7)$ & & $5(20.8)$ & & $3(12.5)$ & & $4(16.7)$ & & $2(8.3)$ & \\
\hline \multicolumn{12}{|l|}{ Sex } \\
\hline Male & 39 & $7(17.9)$ & 0.153 & $9(23.1)$ & 0.762 & $11(28.2)$ & 0.435 & $7(17.9)$ & 1.000 & $7(17.9)$ & 0.473 \\
\hline Female & 21 & $9(42.9)$ & & $6(28.6)$ & & $4(19.0)$ & & $4(19.0)$ & & $2(9.5)$ & \\
\hline \multicolumn{12}{|l|}{ Primary site } \\
\hline node & 54 & $14(25.9)$ & 0.653 & $14(25.9)$ & 1.000 & $14(25.9)$ & 1.000 & $8(14.8)$ & 0.069 & $8(14.8)$ & 1.000 \\
\hline Perpheral node & 6 & $2(33.3)$ & & $1(26.7)$ & & $1(16.7)$ & & $3(50.0)$ & & $1(16.6)$ & \\
\hline \multicolumn{12}{|l|}{ B symptoms } \\
\hline Absence & 37 & $10(27.0)$ & 0.936 & $10(27.0)$ & 0.764 & $11(29.7)$ & 0.283 & $6(16.2)$ & 0.734 & $4(10.8)$ & 0.284 \\
\hline Presence & 23 & $6(26.1)$ & & $5(21.7)$ & & $4(17.4)$ & & $5(21.7)$ & & $5(21.7)$ & \\
\hline \multicolumn{12}{|l|}{ Bulky disease } \\
\hline$<10 \mathrm{~cm}$ & 47 & $11(23.4)$ & 1.000 & $13(27.7)$ & 0.561 & $12(25.5)$ & 1.000 & $8(17.0)$ & 0.552 & $7(14.9)$ & 1.000 \\
\hline$\geq 10 \mathrm{~cm}$ & 4 & $1(25.0)$ & & $1(0)$ & & $1(25.0)$ & & $1(25.0)$ & & $0(0)$ & \\
\hline \multicolumn{12}{|l|}{ Performance status } \\
\hline ECOG 0-1 & 50 & $13(26.0)$ & 1.000 & $13(26.0)$ & 0.965 & $12(24.0)$ & 1.000 & $9(18.0)$ & 0.571 & $8(16)$ & 1.000 \\
\hline ECOG 2-4 & 4 & $1(25.0)$ & & $1(25.0)$ & & $1(25.0)$ & & $1(25.0)$ & & $0(0)$ & \\
\hline \multicolumn{12}{|l|}{ LDH } \\
\hline$<22^{*}$ insitutioal ULN & 30 & $9(30.0)$ & 0.445 & $11(36.7)$ & 0.133 & $9(30.0)$ & 0.445 & $7(23.3)$ & 0.483 & $6(20)$ & 0.277 \\
\hline$>2 *$ insitutioal ULN & 24 & $5(20.8)$ & & $4(16.7)$ & & $5(20.8)$ & & $3(12.5)$ & & $2(8.3)$ & \\
\hline \multicolumn{12}{|l|}{ Extranodal site } \\
\hline$\leq 1$ & 50 & $13(26.0)$ & 1.000 & $14(28.0)$ & 1.000 & $13(26.0)$ & 1.000 & $9(18.0)$ & 0.571 & $8(16)$ & 1.000 \\
\hline$>1$ & 4 & $1(25.0)$ & & $1(25.0)$ & & $1(25.0)$ & & $1(25.0)$ & & $0(0)$ & \\
\hline \multicolumn{12}{|l|}{ Stage } \\
\hline$|/| \mid$ & 29 & $5(17.2)$ & 0.077 & $8(27.6)$ & 0.904 & $10(34.5)$ & 0.168 & $4(13.8)$ & 0.307 & $4(13.8)$ & 1.000 \\
\hline III/IV & 23 & $9(39.1)$ & & $6(26.1)$ & & $4(17.4)$ & & $6(26.1)$ & & $4(17.4)$ & \\
\hline \multicolumn{12}{|l|}{$|P|$} \\
\hline $0-1$ & 30 & $10(33.3)$ & 0.142 & $11(36.7)$ & 0.087 & $11(36.7)$ & 0.037 & $5(16.7)$ & 1.000 & $6(20)$ & 0.269 \\
\hline $2-5$ & 25 & $4(16.0)$ & & $4(16.0)$ & & $3(12.0)$ & & $5(20.0)$ & & $2(8)$ & \\
\hline \multicolumn{12}{|l|}{ Pathological type } \\
\hline GCB & 26 & $4(15.4)$ & 0.084 & $6(23.1)$ & 0.764 & $4(15.4)$ & 0.133 & $3(11.5)$ & 0.320 & $4(15.4)$ & 1.000 \\
\hline non-GCB & 34 & $12(35.3)$ & & $9(26.5)$ & & $11(32.4)$ & & $8(23.5)$ & & $5(14.7)$ & \\
\hline
\end{tabular}

DLBCL diffuse large B-cell lymphoma, GCB germinal center B cell, IPI international prognostic index, $L D H$ lactate dehydrogenase.

In our present study, not only were CNVs detected in clinical sample of DLBCL, but also it were detected in a panel of six cell lines, including DOHH2, OCI-LY1, OCI-LY8, Toledo, Raji, and Namalwa. From the results obtained at the cell-line level as well as in clinical tissues, it can be seen that CNV was a common event in almost all components of the PI3K/AKT signaling pathway.

Using TMA, we found that of all the members subjected to IHC, $\mathrm{p} 110 \alpha, \mathrm{p} 110 \beta, \mathrm{p} 110 \gamma$, and $\mathrm{p} 110 \delta$ protein expression rates were $80 \%, 81.6 \%, 81.6 \%$, and $81.6 \%$, respectively. This was similar to the findings of Meadows's findings in Hodgkin Lymphoma (HL) where they showed that the positive expression of $\mathrm{p} 110 \alpha, \mathrm{p} 110 \beta, \mathrm{p} 110 \gamma$, and p $110 \delta$ was $97.2 \%, 29.2 \%, 54.4 \%$ and $80.6 \%$, respectively [23]. The expression of $p 110 \alpha$ has been reported to be an independent factor for predicting decreased OS for patients with ovarian cancers [17] and gastric cancers [19], although there are no related reports with respect to lymphoma. Protein expressions of $\mathrm{p} 110 \alpha, \mathrm{p} 110 \beta$, 


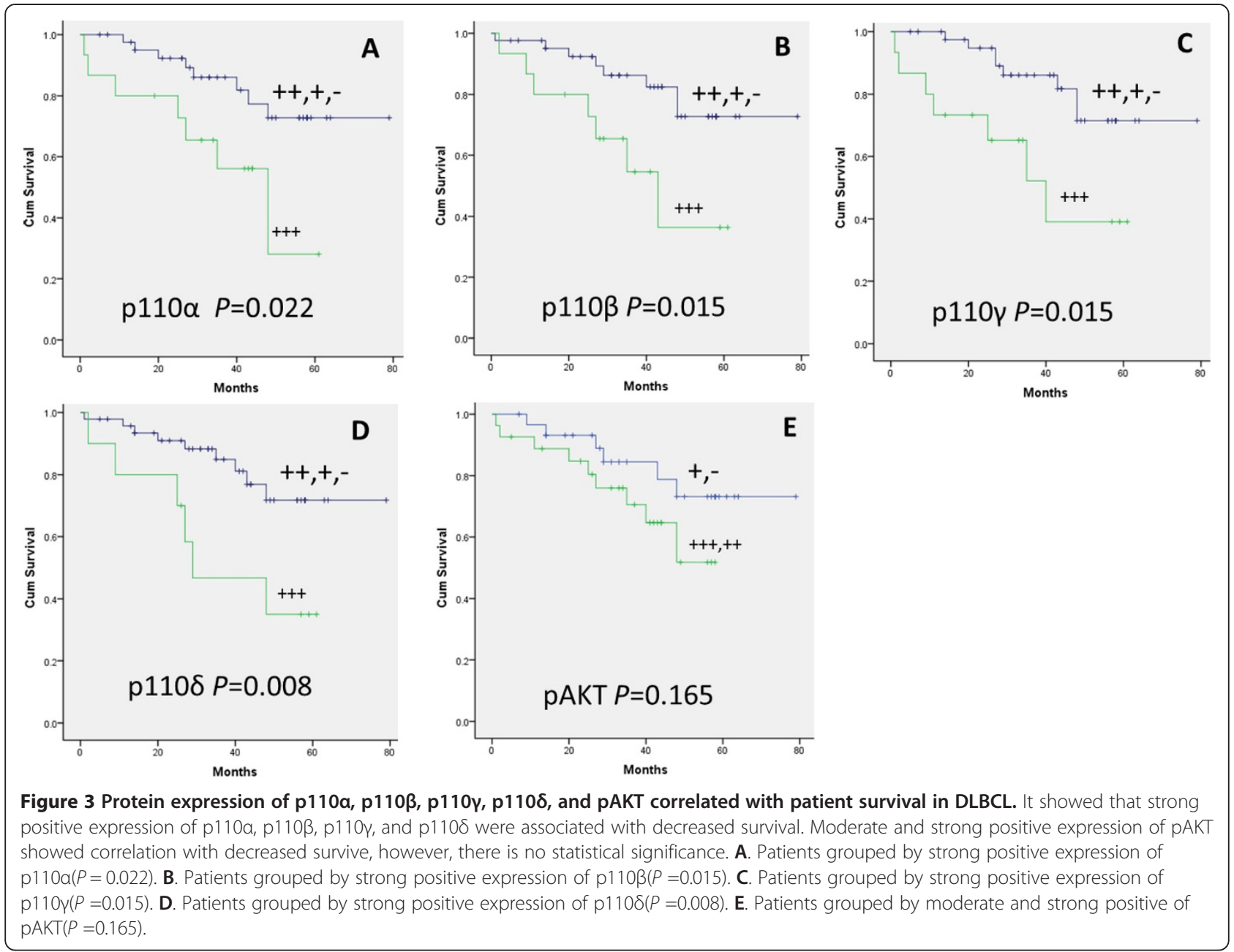

p110y, and p110 $\delta$ showed significant correlation with poor survival.

With regard to AKT, whose active form is phosphorylated AKT (pAKT). It has been reported that $\mathrm{p} 110 \alpha$ amplification was closely associated with pAKT expression. In the study, CNV of PIK3CA was highly associated with aberrant $\mathrm{p} 110 \alpha$ protein expression, which subsequently associated with pAKT, indicating $\mathrm{p} 110 \alpha$ was main isoform for activation of the downstream core protein AKT in DLBCL. PAKT has been extensively reported to be associated with poor prognosis in different types of cancer [15,24-26]. Expression of pAKT has shown a trend towards decreased 5-year survival for patients with DLBCL $(P=0.05)$ [5], while another study showed that high pAKT expression had decreased OS $(P=0.036)$ [27]. In our research, high pAKT expression was associated with poor survival, although statistical significance was not reached. Due to limited number of cases in the study, large cohort study is required to further investigate their relationship and validate our findings.

\section{Conclusions}

In summary, CNVs of PI3K and AKT subunits were a common event in the DLBCL. CNV of PIK3CA is highly associated with aberrant $\mathrm{p} 110 \alpha$ protein expression and subsequent activation of PI3K/AKT pathway. CNVs of PIK3CA and PIK3CB, and aberrant protein expression of p110 isoforms are of great important value for predicting inferior prognosis in DLBCL. Frequent CNVs of PI3K/AKT subunits may play an important role in the tumorigenesis of DLBCL.

\section{Abbreviations}

DLBCL: Diffuse large B-cell lymphoma; GCB: Germinal center B-cell; nonGCG: Non germinal center B-cell; ABC: Active B-cell; IPI: International prognostic index; LDH: Lactate dehydrogenase; TMA: Tissue microarray.

\section{Competing interests}

The authors declared that they have no competing interests.

\section{Authors' contributions}

WC and YC performed experiments and were responsible for data collection, analysis, interpretation of the results, and writing the manuscript. RB and WW were responsible for conducting the data analysis in cooperation with ZL and PW. MS provided clinical samples for performance of experiments. XZ 
were responsible for experimental design, analysis and interpretation. All authors have read and approved the final manuscript.

\section{Acknowledgements}

This work was supported from National Nature Science Funding of China (NSFC) (Code No. 30870985, 30973391 and 81272630). We thank Dr Mingzhi Zhang and Dr B. Hilda Ye for cell lines; Hongyu Gu for immunohistochemistry; Yuhu Xin for technical support during the experiments.

\section{Author details}

${ }^{1}$ Department of Pathology, Fudan University Shanghai Cancer Center, 270 Dong'an Road, Shanghai 200032, PR China. '2Department of Oncology, Shanghai Medical College, Fudan University, Shanghai 200032, PR China. ${ }^{3}$ Institute of Pathology, Fudan University, Shanghai 200032, PR China. ${ }^{4}$ Department of Pathology, The First Affiliated Hospital of Xinjiang Medical University, Urumqi 830054, Xinjiang Uygur Autonomous Region, PR China.

Received: 7 December 2013 Accepted: 6 January 2014

Published: 13 January 2014

\section{References}

1. Swerdlow SH, Campo E, Harri ES, Jaffe ES, Pileri SA, Stein H, Thiele J, Vardiman JW: WHO Classification of Tumours of Haematopoietic and Lymphoid Tissues. Lyon: IARC; 2008.

2. Jemal A, Bray F, Center MM, Ferlay J, Ward E, Forman D: Global cancer statistics. CA Cancer J Clin 2011, 61:69-90.

3. Beer-Hammer S, Zebedin E, von Holleben M, Alferink J, Reis B, Dresing P, Degrandi D, Scheu S, Hirsch E, Sexl V, et al: The catalytic PI3K isoforms p110gamma and p110delta contribute to B cell development and maintenance, transformation, and proliferation. J Leukoc Biol 2010, 87:1083-1095.

4. Engelman JA, Luo J: The evolution of phosphatidylinositol 3-kinases as regulators of growth and metabolism. Nat Rev Gen 2006, 7:606-619.

5. Uddin S, Hussain AR, Siraj AK, Manogaran PS, Al-Jomah NA, Moorji A, Atizado V, Al-Dayel F, Belgaumi A, El-Solh H, et al: Role of phosphatidylinositol 3'-kinase/ AKT pathway in diffuse large B-cell lymphoma survival. Blood 2006, 108:4178-4186.

6. Jia S, Liu Z, Zhang S, Liu P, Zhang L, Lee SH, Zhang J, Signoretti S, Loda M, Roberts TM, Zhao JJ: Essential roles of PI(3)K-p110beta in cell growth, metabolism and tumorigenesis. Nature 2008, 454:776-779.

7. Agell L, Hernandez S, Salido M, de Muga S, Juanpere N, Arumi-Uria M, Menendez S, Lorenzo M, Lorente JA, Serrano S, Lloreta J: PI3K signaling pathway is activated by PIK3CA mRNA overexpression and copy gain in prostate tumors, but PIK3CA, BRAF, KRAS and AKT1 mutations are infrequent events. Mod Pathol 2011, 24:443-452.

8. Psyrri A, Papageorgiou S, Liakata E, Scorilas A, Rontogianni D, Kontos CK, Argyriou P, Pectasides D, Harhalakis N, Pappa V, et al: Phosphatidylinositol 3'kinase catalytic subunit alpha gene amplification contributes to the pathogenesis of mantle cell lymphoma. Clin Cancer Res 2009, 15:5724-5732.

9. Brown JR, Hanna M, Tesar B, Werner L, Pochet N, Asara JM, Wang YE, Dal Cin $P$, Fernandes SM, Thompson C, et al: Integrative genomic analysis implicates gain of PIK3CA at $3 \mathrm{q} 26$ and MYC at 8q24 in chronic lymphocytic leukemia. Clin Cancer Res 2012, 18:3791-3802.

10. Baohua Y, Xiaoyan Z, Tiecheng Z, Tao Q, Daren S: Mutations of the PIK3CA gene in diffuse large B cell lymphoma. Diagn Mol Pathol 2008, 17:159-165.

11. Zhang J, Grubor V, Love CL, Banerjee A, Richards KL, Mieczkowski PA, Dunphy C, Choi W, Au WY, Srivastava G, et al: Genetic heterogeneity of diffuse large B-cell lymphoma. Proc Natl Acad Sci USA 2013, 110:1398-1403.

12. Hans $C P$, Weisenburger $D D$, Greiner TC, Gascoyne RD, Delabie J, Ott G, Muller-Hermelink HK, Campo E, Braziel RM, Jaffe ES, et al: Confirmation of the molecular classification of diffuse large B-cell lymphoma by immunohistochemistry using a tissue microarray. Blood 2004, 103:275-282.

13. Geiss GK, Bumgarner RE, Birditt B, Dahl T, Dowidar N, Dunaway DL, Fell HP, Ferree S, George RD, Grogan T, et al: Direct multiplexed measurement of gene expression with color-coded probe pairs. Nat Biotechnol 2008, 26:317-325.

14. Zhang L, Huang J, Yang N, Greshock J, Liang S, Hasegawa K, Giannakakis A, Poulos N, O'Brien-Jenkins A, Katsaros D, et al: Integrative genomic analysis of phosphatidylinositol 3'-kinase family identifies PIK3R3 as a potential therapeutic target in epithelial ovarian cancer. Clin Cancer Res 2007, 13:5314-5321.
15. Grabinski N, Bartkowiak K, Grupp K, Brandt B, Pantel K, Jucker M: Distinct functional roles of Akt isoforms for proliferation, survival, migration and EGF-mediated signalling in lung cancer derived disseminated tumor cells. Cell Signal 2011, 23:1952-1960.

16. Scrima M, De Marco C, Fabiani F, Franco R, Pirozzi G, Rocco G, Ravo M, Weisz A, Zoppoli P, Ceccarelli M, et al: Signaling networks associated with AKT activation in non-small cell lung cancer (NSCLC): new insights on the role of phosphatydil-inositol-3 kinase. PLoS One 2012, 7:e30427.

17. Huang J, Zhang L, Greshock J, Colligon TA, Wang Y, Ward R, Katsaros D, Lassus H, Butzow R, Godwin AK, et al: Frequent genetic abnormalities of the PI3K/AKT pathway in primary ovarian cancer predict patient outcome. Genes Chromosomes Cancer 2011, 50:606-618.

18. Ma YY, Wei SJ, Lin YC, Lung JC, Chang TC, Whang-Peng J, Liu JM, Yang DM, Yang WK, Shen CY: PIK3CA as an oncogene in cervical cancer. Oncogene 2000, 19:2739-2744

19. Shi J, Yao D, Liu W, Wang N, Lv H, Zhang G, Ji M, Xu L, He N, Shi B, Hou P: Highly frequent PIK3CA amplification is associated with poor prognosis in gastric cancer. BMC Cancer 2012, 12:50.

20. Kreisel F, Kulkarni S, Kerns RT, Hassan A, Deshmukh H, Nagarajan R, Frater JL, Cashen A: High resolution array comparative genomic hybridization identifies copy number alterations in diffuse large B-cell lymphoma that predict response to immuno-chemotherapy. Cancer Genet 2011, 204:129-137.

21. Alizadeh AA, Eisen MB, Davis RE, Ma C, Lossos IS, Rosenwald A, Boldrick JC, Sabet H, Tran T, Yu X, et al: Distinct types of diffuse large B-cell lymphoma identified by gene expression profiling. Nature 2000, 403:503-511.

22. Ye ZQ, Niu S, Yu Y, Yu H, Liu BH, Li RX, Xiao HS, Zeng R, Li YX, Wu JR, Li YY: Analyses of copy number variation of GK rat reveal new putative type 2 diabetes susceptibility loci. PLoS One 2010, 5:e14077.

23. Meadows SA, Vega F, Kashishian A, Johnson D, Diehl V, Miller LL, Younes A Lannutti BJ: PI3Kdelta inhibitor, GS-1101 (CAL-101), attenuates pathway signaling, induces apoptosis, and overcomes signals from the microenvironment in cellular models of Hodgkin lymphoma. Blood 2012, 119:1897-1900.

24. Aleskandarany MA, Rakha EA, Ahmed MA, Powe DG, Ellis IO, Green AR: Clinicopathologic and molecular significance of phospho-Akt expression in early invasive breast cancer. Breast Cancer Res Treat 2011, 127:407-416.

25. Karamitopoulou E, Zlobec I, Panayiotides I, Patsouris ES, Peros G, Rallis G, Lapas C, Karakitsos P, Terracciano LM, Lugli A: Systematic analysis of proteins from different signaling pathways in the tumor center and the invasive front of colorectal cancer. Hum Pathol 2011, 42:1888-1896.

26. Tanaka Y, Terai Y, Tanabe A, Sasaki H, Sekijima T, Fujiwara S, Yamashita Y, Kanemura M, Ueda M, Sugita M, et al: Prognostic effect of epidermal growth factor receptor gene mutations and the aberrant phosphorylation of Akt and ERK in ovarian cancer. Cancer Biol Ther 2011, 11:50-57.

27. Hasselblom S, Hansson U, Olsson M, Toren L, Bergstrom A, Nilsson-Ehle H, Andersson PO: High immunohistochemical expression of p-AKT predicts inferior survival in patients with diffuse large B-cell lymphoma treated with immunochemotherapy. Br J Haematol 2010, 149:560-568.

\section{doi:10.1186/1479-5876-12-10}

Cite this article as: Cui et al:: Frequent copy number variations of PI3K AKT pathway and aberrant protein expressions of PI3K subunits are associated with inferior survival in diffuse large B cell lymphoma. Journal of Translational Medicine 2014 12:10.

\section{Submit your next manuscript to BioMed Central and take full advantage of:}

- Convenient online submission

- Thorough peer review

- No space constraints or color figure charges

- Immediate publication on acceptance

- Inclusion in PubMed, CAS, Scopus and Google Scholar

- Research which is freely available for redistribution 